\title{
Integration of Lightweight Semantic Enterprise Service-Oriented Architecture within Corporate Environmental Management Information Systems
}

\author{
http://dx.doi.org/10.3991/ijes.v1i1.2933 \\ Tariq Mahmoud \\ Carl von Ossietzky University of Oldenburg, Oldenburg, Germany
}

\begin{abstract}
The demand for Corporate Environmental Management Information System (CEMIS) and sustainable development in general requires that decision-makers in all organizational forms are able to take data heterogeneity into account. In this sense, software complexity and integration are considered as important factors to develop such software systems. To handle complexity and integration factors, Service-Oriented Architecture (SOA) supported by sematic enrichment is considered as the most important enabler to address such challenges. This work is devoted to present the main aspects behind the concept of Semantic Enterprise Service-Oriented Architecture (SESOA). The proposed concept tries to overcome data integration problems of the Next Generation CEMIS within the IT-forGreen project. This is achieved in the creation of the project's base component that is the platform. This platform integrates several research concepts of current green interests and tries to investigate their feasibility via prototypical implementations.
\end{abstract}

Index Terms-Web Services, SOA, Semantic Enterprise Service-Oriented Architecture, SESOA, IT-for-Green, Next Generation CEMIS, Green Service Mall.

\section{INTRODUCTION}

Key personnel from the research and transfer network ERTEMIS (European research and transfer network for environmental management information systems) work collaboratively in the IT-for-Green research project to enable organizations and their business processes to become more environmental-friendly in terms of information processing.

In the last decade, research was more concerned about how to integrate different heterogeneous systems by relying on service-orientation concept. SOA paradigm is mainly based on having shared and unique language across different systems to solve the problems of data and process heterogeneity side by side. Web Service-enabled SOA represents one way to realize the service-orientation concept by depending on Web Service as underlying technology.

OASIS SOA Reference Model group had defined SOA as follows: "SOA is a paradigm for organizing and utilizing distributed capabilities that may be under the control of different ownership domains. It provides a uniform means to offer, discover, interact with and use capabilities to produce desired effects consistent with measurable preconditions and expectations" [1].
Web Services are new enablers that promote the possibility of information integration on systems across the borders of organizations and applications. Different distributing technologies had been used to realize SOA. One of the early Web enablement means were the application servers [2]. Using the conventional distributed computing technologies in realizing SOAs in interorganizational scales is not sufficient. This is because these technologies are often centralized and managed by a single company and considered more as intra-enterprise technologies.

When inter-organizational integration perspective is considered, Web technologies standards become more beneficial. These technologies are considered as the essential elements for achieving the integration in Business-to-Business (B2B) environments. Web Services extends the Web enablement and considered as an influential technology to achieve integration by promoting the loose coupling of software functionalities and developing well-defined programmatic interfaces [3].

The annotation of Web Services in SOA-based systems with semantics results in the so-called Semantic Web Services [4], [5]. The main objective behind Semantic Web Services research paradigm is to ease the automation process of Web Services discovery, selection, invocation, composition, and monitoring in open and distributed environments [6].

Semantic Enterprise Service-Oriented Architecture (SESOA) represents a service architecture that enables the provision and usage of Web Services within a distributed network via its semantic service repository. This approach is designed and developed to be responsible of splitting the semantic annotation from the description of core services where both Web Services and Semantic Web Services can be used, evaluated and validated [7].

SEOSA represents a competent nominee to realize Next generation CEMIS within the IT-for-Green European project (further information about this project is available at: http://www.it-for-green.eu/). The demands for CEMIS and sustainable development require the ability of decision-makers in all organizational forms to take a variety of heterogeneous data into consideration. The factors that impact the development of sustainable products, business processes or holistic models for causeand-effect chains are still missing or incomplete. A resource-friendly design of business processes and their energy- and material-efficient will control additional demands for sustainability-oriented organizational structures. 
IT-for-Green research project tries to address the main challenges related to the environmental aspects by developing three modules integrated by a service-oriented platform. The main characteristics of this platform are derived from SESOA. This paper gives overview about SESOA and how it supports the development of Next Generation CEMIS in IT-for-Green project. This is done in the creation of its base component that is the serviceoriented platform. This platform integrates research concepts of current green interests and investigates their practicability through prototypical implementations.

The rest of this paper is constructed as follows: Section Two gives a short description of the IT-for-Green research project and its modules. It is followed by the presentation of SESOA in Section Three with some insights of how the semantic annotation of Web Services is provided. The main aspects transferred from SESOA to the IT-for-Green core service-oriented platform are then depicted in Section Four. Section Five shows the importance of this work in comparison with related work. The paper then concludes with brief summary of the contributions behind this paper.

\section{IT-FOR-GREEN RESEARCH PROJECT}

To overcome today's environmental challenges, the European Research and Transfer Network for Environmental Management Information Systems (ERTEMIS) had been set up (more information is available at: http://www.ertemis.eu/). This network initiates the "IT-for-Green: Environment, Energy and Resource Management with Next Generation CEMIS" research project in 2011. The main goal behind this project is the development of a CEMIS that covers the whole product lifecycle. The key components of this CEMIS are included in its three modules, namely "Green IT", "Green Logistics" and "Sustainability Reporting and -dialogue". Module 1 or the "Green IT" captures and automatically measures energy consumption of different ICT realized by data centers. Module 2 or the "Green Logistics and Sustainable Product Development" provides functions for automatic determination of $\mathrm{CO}_{2}$-emissions along the supply chain. Module 3 or "Sustainability Reporting and Communication" sets up a Web-based reporting solution using the SOA paradigm. Additionally a service-oriented platform that integrates these three modules is provided. This platform is designed to be open and extensible for new modules and services through workflow-based and Web Service-enabled SOAs [8].

Sometimes it is too late to set the right course at the time of reporting environmental performance. Without an early cause and an effect identification to predict environmental effects of decisions for timely intervention, potentials for pre-cautious remain unemployed. To handle these challenges and to map the whole lifecycle of a product, new workflow engines for the Next Generation CEMIS are needed [9]. The product's lifecycle starts out from input (measuring energy efficiency of the used ICT and production schemes), goes on with transformation processes (environmental conscious production, green logistics and sustainable product development) and ends up at the output stage (business communication and sustainability reporting) and even beyond (disassembling planning). These workflow engines represent the core of the IT-for-Green platform's component [10]. As mentioned before, new workflow engines need to be utilized in order to handle all business processes through any product's lifecycle. The design and utilization of such workflow engines will support but not limited to:

- Long product life, i.e. accompany a product through the whole life cycle

- Capability of flexibly integrating (almost) arbitrary activities

- Integration of overall CEMIS contexts for standardized information exchange and integration

- Capability of frequently interrupting and resuming execution at arbitrary breakpoints

- Integration of freely programmable control-flow structures

- Redefinition of transitions and states and integration of new activities at runtime (for long living workflows)

The underlying architectures of such engine enable a collection of green Web Services. In the future, they will provide the basis facilities for a service registry to be included within the project's service-oriented platform (called Green Service Mall). To succeed in implementing the Next Generation CEMIS, the experience and skills of different scientific and industry partners is combined.

\section{SEMANTIC-ENABLED ENTERPRISE SOA}

Based on [11], SESOA framework can be defined as: "an enterprise solution that links businesses to external systems based on business process and service orientation concepts. It represents a lightweight Web framework that annotates Web Services coming from different service providers with semantics so that the indexing and discovery of these services can be more comprehensive". Examples of external service providers can be those who provide Web Services for product shipment or payment in the domain of enterprise information systems. Other examples are the providers who provide Web Services to measure environmental indicators, carbon footprints or $\mathrm{CO}_{2}$ emissions in the domain of environmental management information systems. Using the concepts derived from SESOA, these service providers can publish their services in a common registry called the semantic service repository. Therefore, SESOA can be considered as an enhanced SOA concept in which any Web Service consumer can discover its semantic service repository and invoke the provider's services.

Moreover, SESOA supports the traditional SOA paradigm and offers also the traditional discovery possibilities provided by former techniques like the Universal Description Discovery and Integration (UDDI) standard. The research process that has been followed to design SESOA is derived from the design science research methodology proposed by [12] and the design science in in-formation systems research proposed by [13].

\section{A. Semantic Support of Web Services}

The Web Services advertised in this semantic service repository are semantically annotated using Resource Description Framework (RDF) statements. These statements are merely subject-predicate-object statements that link the services in groups called assemblages. The subject in such statement is for example the assemblage itself, the predicate is the relationship like "hasMember", and the object is the Web Service itself. 


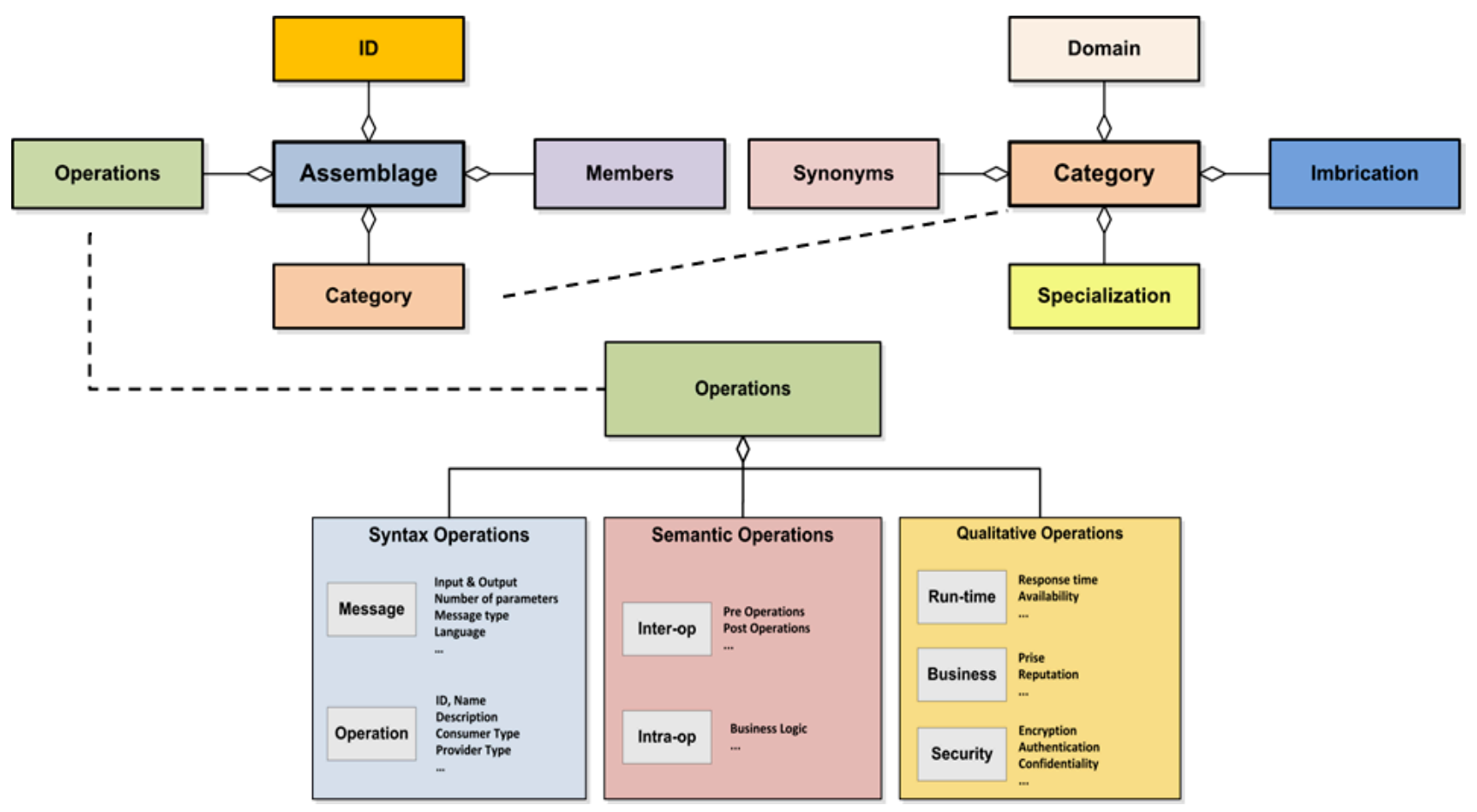

Figure 1. SESOA Semantic Support of Web Services

In this way, any Web Service can be semantically queried based on the assemblage to whom it belongs by utilizing any RDF query language like SPARQL Protocol and RDF Query Language (SPARQL) [14].

An assemblage is a holder or mini repository that groups Web Services coming from different providers. This grouping is based on the business sectors or domains to which these services usually belong. The internal structure of assemblages is depicted in Fig. 1.

Each assemblage has an ID, a category, members and operations. The $I D$ is an identifier that contains a unique name and a text description. It indicates the assemblage's characteristics. The category specifies the assemblage's classification within a specific business domain. All Web Services that belong to one assemblage have the same category. The categories are accessible to all service providers in order to let them registering their services within it. Being member of an assemblage, service providers assure that their Web Services are registered in the respected assemblages in the members list. Finally, the operations (properties) are abstract operations that depict the main functions that the assemblage's members have to specify. The Web Service developer in the proposed architecture has the duty of defining these operations. They can be classified into either syntax, semantic or qualitative operations.

The above-mentioned internal structure of the assemblages has some similarity with the "communities" proposed by [15]. While these "communities" serve in his work to facilitate the dynamic service composition, assemblages in this work are more related to the design aspects in classifying services based on the business domain to which they belong.

Fig. 1 shows also that each assemblage's category has four properties: domain, synonyms, specialization, and imbrications. A category's domain represents the main assemblage's business area (e.g., Green IT). It is possible that different assemblages adopt different taxonomies to define their category attribute. Synonyms, the second category's property, contain similar or optional domain names for an assemblage. For example, "energy consumption, energy measurement" are synonyms of "green IT". The third category's property is the specialization that is a set of assemblage's domain characteristics. For example, "data center" and "ICT" are specializations of "Green IT". This means that this assemblage provides services for both types. The last category's property is the imbrications. Since assemblages can generally have something in common, they are linked to each other through relationships specified in the imbrications attribute. It contains the list of assemblages' categories that overlap with each other. For example, an operation that belongs to an assemblage with the domain "Green IT" might relate to another operation that belongs to an assemblage with the domain "Sustainability Reporting". This provides energy measurement services for sustainability reporting. The Web Service developers are responsible of identifying related categories to assign them to the imbrications property.

Finally, Fig. 1 shows that each assemblage has a set of operations. As mentioned before, these operations are classified into syntax, semantic, and qualitative operations. Each of these classes is specified separately. For the syntactic and semantic operations, they are classified into messages, operations and as inter- and intra-operations respectively. As for the qualitative operations, they include general runtime, business, security and privacy information. 


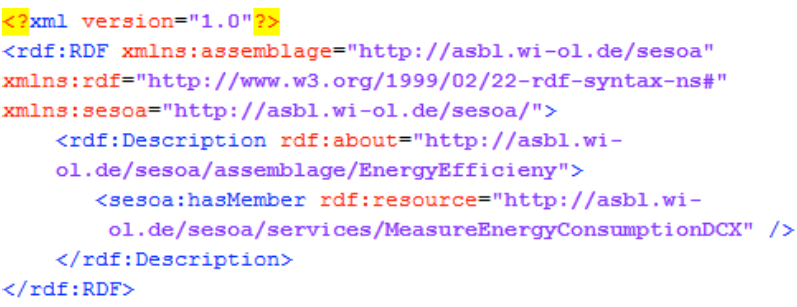

Figure 2. RDF Statement Example

To sum up, Each Web Service is a member of one or more assemblages. The relationships between services and assemblages are semantically annotated using RDF statements. Each RDF statement is realized as a subjectpredicate-object triple in which the subject is the assemblage, the predicate is the relationship (e.g. "hasMember") and the object is the Web Service. Each of these parts can be identified using a distinct URI. Fig. 2 represents an example of an RDF statement between an assemblage called "energy efficiency" and one of its services called "measure energy consumption in data centre $\mathrm{x}$ ".

These RDF statements are published in the system's semantic service repository in which any RDF query language like SPARQL can be used to query services. An important point that has to be taken into consideration in this context is that the semantic querying in this system takes place at the workflow management system level in which business processes are constructed. This ensures the dynamicity and consistency of the system and opens the possibilities to include up-to-date services in the everchanging business processes.

\section{B. System Components and their Interactions}

In SESOA, all business processes are realized using workflows and most business functionalities are realized using Web Services.

These services can be invoked by any service consumer while executing the system's workflows. Moreover, the main characteristics of this system are:

- It is a process-oriented system in which business processes are managed, implemented, and executed using Web Services;

- It validates Web Services following predefined businesses' criteria, evaluates them using a machine-to-machine evaluation protocol;

- It represents a lightweight semantic-enabled SOA solution;

- It groups Web Services based on the business domains to which they belong;

- It provides semantic annotations to the Web Services relations using RDF statements;

- It supports service reuse because of the adoption of the SOA concept.

Fig. 3 depicts the reference architecture of SESOA. Provider system, consumer system, and Web Service directory are the main components of the SOA paradigm. The core component in this architecture is the processing system. It is a workflow-based system. It follows the recommendation of the workflow reference model proposed by the Workflow Management Coalition
(WfMC) [16]. From this component, all business processes can be executed in form of workflows with connection to the other components of the reference architecture.

Web Services, as mentioned before, are grouped in assemblages based on their business domain. The assemblage unit within the semantic Web Service-based system component in the SESOA reference architecture is responsible of managing these assemblages. This management includes the creation, updating and deletion of assemblages. Detailed information about the internal architecture of the assemblages is provided in the next section.

All Web Services are registered as members in one or more assemblages. The relations between services and assemblages are semantically annotated using RDF statements. These statements are made available in the semantic service repository component. All RDF statements, business and user management data are stored in the SESOA database system.

The validation system has four subsystems namely: ranking unit, annotation provider unit, announcement unit, and service test unit. The ranking unit subsystem is responsible of evaluating the services supplies by external service providers (provider system) using a machine-tomachine evaluation protocol. This protocol is designed based on two criteria namely response time and availability of Web Services. The annotation provider unit is responsible of annotating the relations between the services and the assemblages using RDF statements. Moreover, it stores these relations in the SESOA database system. The announcement unit advertises or makes announcements regarding new service functionalities which external service providers may implement. Finally, the service test unit subsystem is responsible of providing a set of tests exposed as Web Services like primitive data types tests (integer, string, decimal...).

The main interactions between SESOA components are explained as follows [11], [17]:

- Consumer system starts to run one of the activities within the processing system via the system's front end.

- The processing system forwards this request to the assemblage unit within the semantic Web Service-based system to check whether there is a corresponding service or not.

- The semantic Web Service-based system calls the semantic service repository to see in which assemblage the service(s) are located.

- Upon finding corresponding service(s), it will respond back to the consumer system via the processing system.

- Direct binding will be then made available between the consumer system and the service(s).

- After that and at the end of executing the system's workflows, the services, supplied by the external service providers, are validated and evaluated (ranked) using the validation system.

The following section will show the platform of the Next generation CEMIS and how CEMIS is supported by some of SESOA components. 


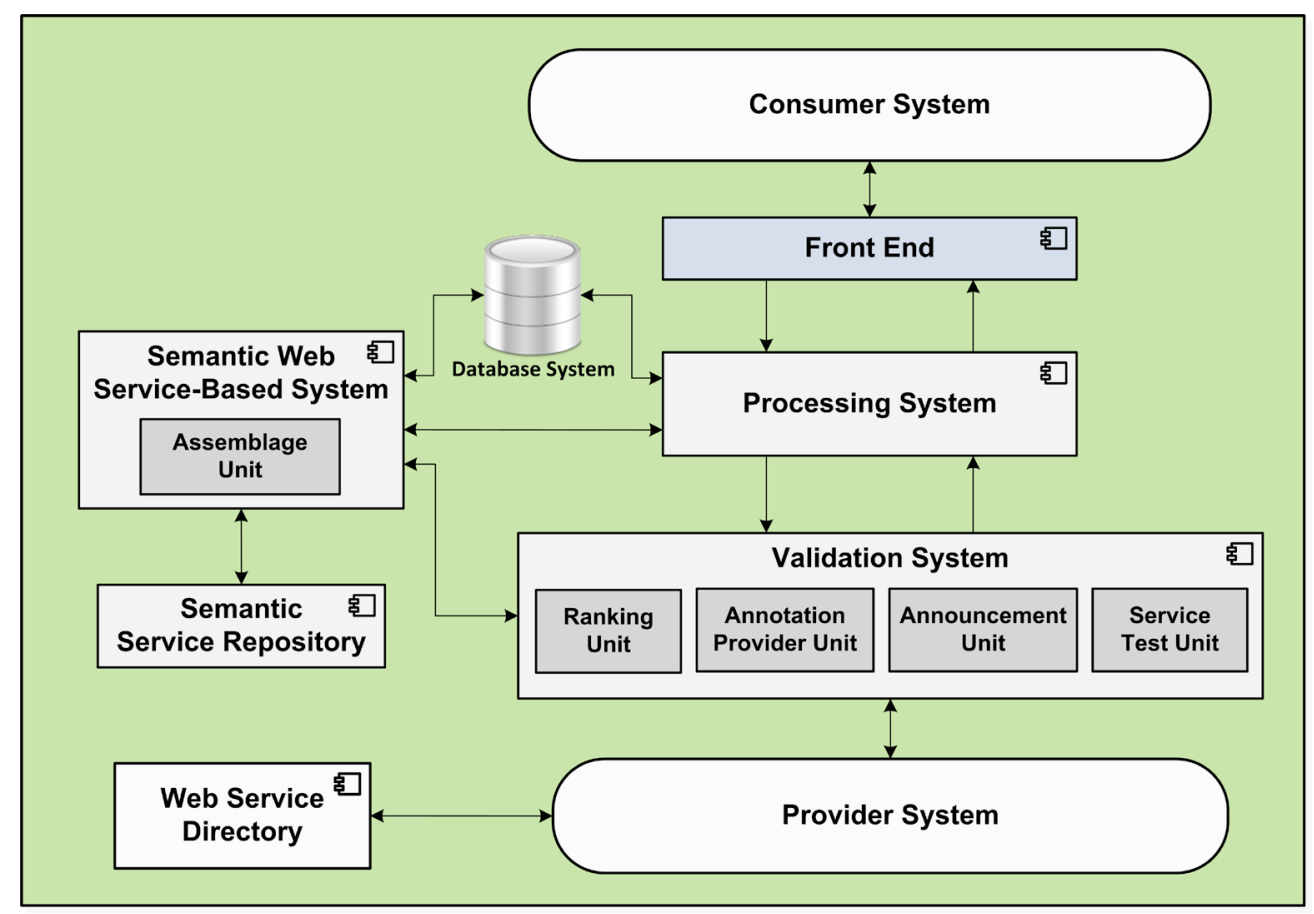

Figure 3. The SESOA Reference Architecture [7], [11]

\section{CEMIS SUPPORTED BY SESOA}

SESOA reference architecture has been investigated to answer the following question: what are the proper components that can be transferred into the Next Generation CEMIS architecture? Upon defining the general requirements for the IT-for-Green project, it has been decided by the team members to adapt some of the components of the SESOA reference architecture to design the project's service-oriented platform (the running environment). These components include: the consumer system, the provider system and the service repository (the Green Service Mall). Moreover, a workflow management system will be applied to the Next Generation CEMIS. The difference between Next Generation CEMIS and SESOA regarding the workflow management system is that an open source workflow system will be applied to the Next generation CEMIS instead of the Microsoft Workflow Foundation that is utilized in SESOA.

It had been also decided that all the business processes included in the resulted software from the project will be implemented as workflows with an environmental context. These workflows are designed to include activities to be implemented as Web Services. These Web Services will be published in the system's repository that is called "Green Service Mall".

From a technical perspective, the development of the needed services for realizing an activity is the main concern in the platform of the Next Generation CEMIS. This is seemingly the business logic of the overall system. For the sake of an individually composed software system, that serves exactly the individual needs of different business and companies, a set of interoperable services for individual tasks are to be developed. With these modular design principles, each enterprise or organization may customize its own tailored CEMIS.

In the IT-for-Green CEMIS platform, a workflow represents an executable arrangement of different activities that guides a user during her/his work with the system. It will be possible to define (or rather implement) complex relations and control flows within a workflow. A workflow may comprise activities from several (or even external) sources and also from different users. On a time scale, a workflow might be executed in several phases (during each product lifecycle) and can be saved physically in the meantime. In order to share information among all these services and phases, a specialized context for information exchange is shared [10].

Fig. 4 depicts the service-oriented platform of the Next Generation CEMIS in the IT-for-Green project. This CEMIS is built in a modular way whereby serviceorientation concept is considered as the major conceptual design. Service-orientation means in this context that the smallest units of the CEMIS modules can be realized as Web Services (called Green Web Services). These services are published then in the so-called Green Service Mall (service registry). Following service-orientation paradigm will improve the system's functionality via the integration of new or modified services.

The runtime environment is the central element of the Next Generation CEMES. It is made available on a dedicated Web Server. Fig. 4 shows the architecture of the service-oriented platform with a focus on this runtime environment. Primarily, it provides possibilities to integrate new services through Web Service provision (1) besides modeling (2) and execution (4) of workflows. The workflow execution is managed by a workflow engine. 
PAPER

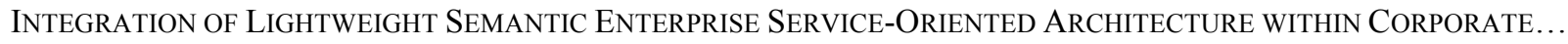

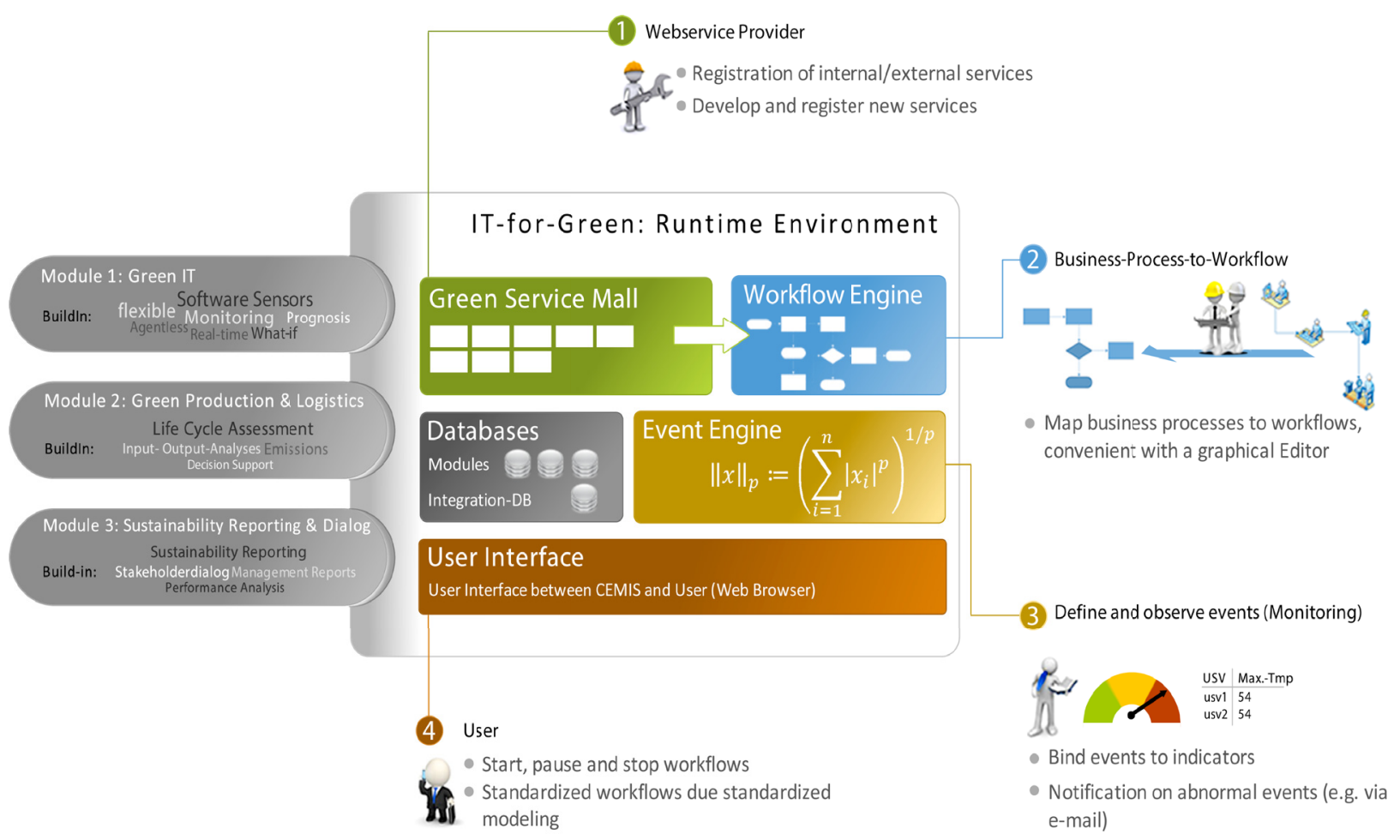

Figure 4. IT-for-Green: The Platform of the Next generation CEMIS [7]

Using such engine (graphical user interface) enables the internal business processes of the organization to discover and invoke the Green Web Services that are published in the Green Service Mall. The workflow editor makes use of the Web Services registered in the Green Service Mall without taking into consideration their origin whether they have been created externally or internally within the company. Web Service providers can be either external entities or internal providers who develop customized services and make it available by publishing it in the Green Service Mall. The specific metric events within the runtime environment can be defined using the Event Engine (number 3 in the figure below). Temperature excess of a threshold value in a date center is a typical example of such events.

The Green Service Mall is the central part of the service-oriented platform. This component provides a set of "yellow pages" and makes them available on the Web. All the services developed in the three modules of Next Generation CEMIS are advertised as services in this mall. The workflow editor can then orchestrate different workflows by discovering, selecting, and invoking these services. In summary, the Green Service Mall supports all phases of any Web Service starting from the discovery till the invocation phase.

The user interface is called by system's users through any Web browser. In particular, the execution of workflows is done starting from this point. Workflows can directly be started, paused and stopped from a Web browser. Another advanced part of the user interface is a customized dashboard responsible of monitoring the defined events besides the classification provided at the
Green Service Mall (presented as a catalog of services and groups of services classified based on specific criteria).

Authorizations for specific functions and areas can be granted using the rights and roles system that is responsible of issuing rights and roles for services. The rights and role system does not only restrict the access to individual services, rather it hides the information from all unauthorized users (or groups).

The development of the Next Generation CEMIS is realized as a Web application that can be used to build on top of it several business scenarios. Table I shows an example of a fictional energy consumption measurement. It calls the responsible Web Service and returns the measurement number, the date, and the status of this measurement. Status can be either over limit, below limit or normal. This status attribute is essential and sometimes crucial for the system's developers to maintain the energy consumption values. This example is one of many examples of the event engine subsystem of the serviceoriented platform [17].

TABLE I.

ENERGY CONSUMPTION MEASUREMENT EXAMPLE

\begin{tabular}{|l|l|l|l|}
\hline Measurement number & Date & Status & \\
\hline 92 & $20 / 05 / 2013$ & Normal & Select \\
& $3: 42: 56$ PM & & \\
\hline 91 & $18 / 05 / 2013$ & Normal & Select \\
\hline 90 & $4: 17: 14$ PM & & Select \\
\hline 89 & $18 / 05 / 2013$ & Over Limit & Select \\
& $4: 15: 06$ PM & & Normal \\
\hline
\end{tabular}




\section{EVALUATYON}

Brief analysis of the funded projects in the domain of Corporate Environmental Management from the last fifteen years has indicated that there is a strong need for research in the contexts of society, politics, business and science. None of these projects had the objective of providing support to all parts of a product's environmental management lifecycle. Moreover, a cross-corporate review on the sustainability factor of the whole supply chains (sustainable supply chains) has been a casual topic in scientific literature but until now, it has not found its way to business practice.

In contrast to such projects, ERTEMIS and IT-forGreen project will emphasize exactly the aspects of strategic level integration and sustainability needs of the Next Generation CEMIS. Based on [8], The new resulted system with its prototypes will have many added values to business users by enabling them to:

- Develop environmental production and reverse logistics processes;

- Develop hybrid products (integrated services and associated services) taking into account sustainability ideas to open up new markets for sustainable products;

- Realize an interactive exchange of information between different stakeholders in the field of sustainability reporting [18] based on new internet technologies (blogs, wiki, Semantic Web, podcasts, etc.);

- Realize synergy effects, cost cutting effects, strategic advantages, etc. by offering or using Green Web Services from the Green Service Mall or based on service-oriented architectures.

- Pinpoint complementary cause-effect-relations and their effects on different targets from a strategic perspective.

Whereas traditional CEMISs might be considered more as isolated- and functional-oriented information systems (cf. [19]), Next Generation CEMIS will take a holistic approach that guides organizations to a strategic orientation. In this way, the new system is considered more as an information system that deals with material and energy efficiency, emission and waste minimization, reverse logistics, stakeholder support, legal compliance and especially strategic environmental management. As yet, such systems only exist as concepts within scientific discussions. Another important point to be mentioned hers is that there is a strong need for research and knowledge transfer especially in SME market to be identified as an important prerequisite for putting such systems into production.

A ubiquitous ICT and continuously integration of digital and physical systems allow for a fundamental renewal of business processes regarding sustainable business development, for an increased transparency as well as for a better control of material and energy usage.

In order to map the whole lifecycle of a product, the three aforementioned modules in the new system must be designed and developed based. These modules represent the reference implementation and the proof of concept for the Next Generation CEMIS within the IT-for-Green project.

\section{CONCLUSION}

Next Generation CEMIS opens a new way of defining environmental management-related applications on demand from an easily available and externally (via experts) updated toolbox of remote services bundled together with in-house processes and data. This paper presented one of the first considerations regarding the new way of implementing future CEMIS based on the architectural principles harvested from SESOA. Such consideration will redefine workflows as executable state machine definition with the ability of integrating different scripting languages as well as extensible mark-up definitions and activity descriptions.

A standardized, common data format accompanies the workflow definition. In this way, the resulted system will facilitate an easy implementation by definition approach of future highly integrative Next generation CEMIS.

Furthermore, this paper shows that SESOA components had been seen as basis to create the Next generation CEMIS's service-oriented platform that integrates the system's three modules. The resulted prototypical implementation from the IT-for-Green project will enable its users, based on their rights and roles, to access its Green Service Mall to discover, select, and invoke its Green Web Services.

The user management has been considered as a clear requirement in designing the system's database schema. The semantic enablement derived from the SESOA reference architecture might form a good part of the future development of the prototypes resulted from the IT-forGreen project. Therefore, the RDF semantic enrichment of the resulted software from this project is seen as a potential outlook for a future extension of the Next Generation CEMIS.

\section{ACKNOWLEDGMENT}

This work is part of the project IT-for-Green (Next Generation CEMIS for Environ-mental, Energy and Resource Management). The IT-for-Green project is funded by the European regional development fund (grant number W/A III 80119242).

\section{REFERENCES}

[1] C. M. MacKenzie, K. Laskey, F. McCabe, P. F. Brown, and R. Metz, "Reference Model for Service Oriented Architecture 1.0," OASIS | Advancing open standards for the information society, 2006. [Online]. Available: docs.oasis-open.org/soa-rm/v1.0/soarm.doc. [Accessed: 29-Aug-2012].

[2] D. Oberle, "Semantic Management of Middleware," University of Karlsruhe (TH), Karlsruhe, Germany, 2005.

[3] S. Lamparter, "Policy-based Contracting in Semantic Web Serivce Markets," University of Karlsruhe (TH), Karlsruhe, Germany, 2007.

[4] R. Studer, S. Grimm, and A. Abecker, Semantic Web Services: Concepts, Technologies, and Applications. Heidelberg, Germany: Springer Verlag, 2007. http://dx.doi.org/10.1007/3-540-70894-4

[5] S. A. McIlraith, T. C. Son, and H. Zeng, "Semantic Web Services," Intell. Syst. Ieee, vol. 16, no. 2, pp. 46-53, 2001. http://dx.doi.org/10.1109/5254.920599

[6] T. Payne and O. Lassila, "Semantic Web Services," Intell. Syst. Ieee, vol. 19, no. 4, pp. 14-15, 2004. http://dx.doi.org/10.1109/ MIS.2004.29

[7] T. Mahmoud, "CEMIS Next Generation Supported by Semantic Enterprise Service-Oriented Architecture," in Proceedings of the 5th International Conference on Web and Information Technologies, Hammamet, Tunisia, 2013, pp. 21-30. 
PAPER

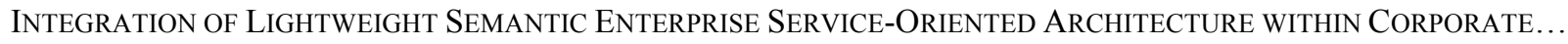

[8] B. Rapp, A. Solsbach, T. Mahmoud, A. Memari, and J. Bremer, "IT-for-Green: Next Generation CEMIS for Environmental, Energy and Resource Management," in EnviroInfo 2011 Innovations in Sharing Environmental Observations and Information, Ispra, Italy, 2011, pp. 573-581.

[9] B. Rapp, J. Vornberger, F. Renatus, and H. Gösling, "An Integration Platform for IT-for-Green: Integrating Energy Awareness in Daily Business Decisions and Business Systems," in Proceedings of the 1st International Conference on Smart Grids and Green IT Systems, Porto, Portugal, 2012, pp. 226-231.

[10] J. Bremer, T. Mahmoud, and B. Rapp, "Implementing CEMIS Workflows with State Chart XML," in EnviroInfo 2012 - Part 2: Open Data and Industrial Ecological Management, Dessau, Germany, 2012, pp. 749-757.

[11] T. Mahmoud, M. Petersen, and D. Rummel, "Business Process Integration within Lightweight Semantic-Enabled Enterprise Service-Oriented Architecture," in E-Procurement Management for Successful Electronic Government Systems, P. O. de Pablos, J. M. Cueva Lovelle, J. E. Labra Gayo, and R. Tennyson, Eds. EProcurement Management for Successful Electronic Government Systems, 2012, pp. 181-192. http://dx.doi.org/10.4018/978-14666-2119-0.ch012

[12] K. Peffers, T. Tuunanen, M. Rothenberger, and S. Chatterjee, "A Design Science Research Methodology for Information Systems Research," J. Manag. Inf. Syst., vol. 24, no. 3, pp. 45-77, 2008. http://dx.doi.org/10.2753/MIS0742-1222240302

[13] A. R. Hevner, S. T. March, J. Park, and S. Ram, "Design Science in Information Systems Research,” Mis Q., vol. 28, no. 1, pp. 75105, 2004.

[14] E. Prud'Hommeaux and A. Seaborne, "SPARQL Query Language for RDF," W3C Recommendation, 2008. [Online]. Available: http://www.w3.org/TR/rdf-sparql-query/. [Accessed: 09-Aug2011].
[15] B. Medjahed, "Semantic Web Enabled Composition of Web Services," $\mathrm{PhD}$ Thesis, Virginia Polytechnic Institute and State University, Falls Church, Virginia, USA, 2004.

[16] D. Hollingsworth, "Workflow Management Coalition: The Workflow Reference Model," Work. Manag. Coalit., 1995.

[17] T. Mahmoud, J. Marx Gómez, A. Rezgui, D. Peters, and A. Solsbach, "Enhanced BI Systems with On-Demand Data Based on Semantic-Enabled Enterprise SOA," in Proceedings of the 20th European Conference on Information Systems, Paper 184, Barcelona, Spain, 2012.

[18] R. Isenmann and J. M. Gómez, Internetbasierte Nachhaltigkeitsberichterstattung: Maßgeschneiderte StakeholderKommunikation mit IT. Erich Schmidt Verlag GmbH \&, 2008.

[19] O. El-Gayar and B. D. Fritz, "Environmental management information systems (EMIS) for sustainable development: A conceptual overview," Commun. Assoc. Inf. Syst., vol. 17, no. 1, p. 34, 2006.

\section{AUTHORS}

Tariq Mahmoud had studied Information Engineering at Al-Baath University (Homs, Syria) from 2000 till 2005. He was also a research assistant at Al-Baath University till 2007 where he started to conduct a PhD degree at Carl von Ossietzky University of Oldenburg, Germany. He is currently a research fellow at the working group of business information systems at the Carl von Ossietzky University of Oldenburg (Germany). His research work focuses on applying semantics to SOA-enabled enterprise solutions, information security, and Semantic Web

Submitted 20 June 2013. Published as re-submitted by the authors 23 July 2013. 